EPJ Web of Conferences 86, 00064 (2015)

DOI: $10.1051 /$ epjconf/ 20158600064

(C) Owned by the authors, published by EDP Sciences, 2015

\title{
Low energy incomplete fusion and its relevance to the synthesis of super heavy elements
}

\author{
Abhishek Yadav, a , P Kumar², A Raghav², Mohd Shuaib², V R Sharma², D P Singh ${ }^{3}$, P P Singh ${ }^{4}$, Sunita Gupta $^{5}$, U

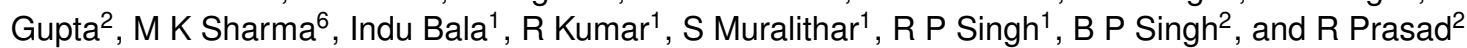 \\ ${ }^{1}$ NP Group: Inter-University Accelerator Centre, Aruna Asaf Ali Marg, New Delhi-110 067, Delhi, India \\ ${ }^{2}$ Department of Physics, Aligarh Muslim University, Aligarh-202 002, Uttar Pradesh, India \\ ${ }^{3}$ Department of Physics, University of Petroleum and Energy Studies, Dehradoon-248 007, Uttrakhand, India \\ ${ }^{4}$ Department of Physics, Indian Institute of Technology Ropar, Roopnagar-140 001, Punjab, India \\ ${ }^{5}$ Department of Physics, Agra College, Agra-282 001, Uttar Pradesh, India \\ ${ }^{6}$ Department of Physics, S. V. College, Aligarh-202 001, Uttar Pradesh, India
}

\begin{abstract}
To study the presence of incomplete fusion at energies around the Coulomb-barrier and to understand its dependence on various entrance-channel parameters, the incomplete fusion fractions have been deduced $(i)$ from excitation function measurements for ${ }^{18} \mathrm{O},{ }^{13,12} \mathrm{C}+{ }^{159} \mathrm{~Tb}$, and (ii) from forward recoil range measurements for ${ }^{12} \mathrm{C}+{ }^{159} \mathrm{~Tb}$ systems, at low energies $(<7 \mathrm{MeV} / \mathrm{A})$. The data have been analyzed within the framework of compound nucleus decay, which suggests the production of $x n / p x n$-channels via complete fusion, as these are found to be well reproduced by PACE4 predictions, while, a significant enhancement in the excitation functions of $\alpha$-emitting channels has been observed over the theoretical ones, which has been attributed due to the incomplete fusion processes. Further, the incomplete fusion events observed in case of forward recoil ranges have been explained on the basis of the breakup fusion model, where these events may be attributed to the fusion of ${ }^{8} \mathrm{Be}$ and/or ${ }^{4} \mathrm{He}$ from ${ }^{12} \mathrm{C}$ projectile to the target nucleus. For better insight into the underlying dynamics, the deduced fractions of incomplete fusion have been compared with other nearby systems as a function of various entrance channel parameters. The incomplete fusion has been found to be sensitive to the projectile's energy and alpha-Q-value of the projectile.
\end{abstract}

\section{Introduction}

In super-heavy element (SHE) production laboratories, considerable efforts are being employed to synthesize the SHE using heavy-ion induced complete fusion (CF) reactions with low excitation energy [1]. In addition to the fission and quasi-fission, the existence of incomplete fusion (ICF) at low incident energies (i.e., $\approx 4-7 \mathrm{MeV} / \mathrm{A}$ ) may add complexity to the synthesis of SHEs. In general, at these energies, CF is supposed to be the sole contributor to the total fusion cross section [2]. However, in recent years a large fraction of ICF has been observed at energies as low as $\approx 4-7 \mathrm{MeV} / \mathrm{A}[3-7]$. The onset of ICF at near barrier energies triggered the resurgent interest to understand the ICF reaction dynamics. In ICF reactions the incident projectile is assumed to break up into its fragments, as a consequence of excess input angular momentum, and one of the breakup fragments fuses with the target nucleus [8]. It is customary to disentangle CF and ICF on the basis of driving angular momenta ( $\ell$-values) [8]. At central and/or near-central interactions for $\ell$-window $0 \leq \ell \leq \ell_{\text {crit }}$, the CF is expected to be the dominant process, where intimate contact and transient amalgamation of entire projectile and target nucleus leads to the formation of an excited compound nucleus with pre-determined charge/mass and excitation energy. However, for peripheral collisions or at sufficiently higher energies, $\ell$-values may be higher than $\ell_{c r i t}$. As a consequence fusion of entire projectile may be hindered and gives way to the ICF. This forms an incompletely fused composite (IFC) system, and direct projectile-like-fragments (PLFs) found to be centered in the forward cone. This IFC have relatively less mass/charge and excitation energy (due to partial fusion of projectile), but have high angular-momenta (imparted due to non-central interactions) as compared to the $\mathrm{CN}$ formed via $\mathrm{CF}$ [8]. The additional break-up degrees of freedom make the fusion process more complicated and the possible reaction processes may be; $i$ ) the non-capture break-up, when none of the breakup fragments is captured, ii) ICF, when one of the breakup fragments is captured, iii) sequential complete fusion ( $\mathrm{SCF}$ ), the successive capture of all fragments by the target nucleus. Experimentally, it is not possible to disentangle the cross-sections for direct $\left(\sigma_{D C F}\right)$ and sequential $\left(\sigma_{S C F}\right)$ complete fusion, because both channels lead to the same final reaction residues. Hence, the CF cross-section $\left(\sigma_{C F}\right)$ is taken as the sum of $\sigma_{D C F}$ and $\sigma_{S C F}$, whereas the sum of $\sigma_{C F}$ and incomplete

\footnotetext{
a e-mail: abhishekyadav117@gmail.com
} 
fusion cross-section $\left(\sigma_{I C F}\right)$ may be referred to as the total fusion cross-section $\left(\sigma_{T F}=\sigma_{C F}+\sigma_{I C F}\right)$. After the first experimental observation of "fast $\alpha$-particles" at energies $\approx 10.5 \mathrm{MeV} / \mathrm{A}$ [9], a variety of experimental and theoretical studies have been devoted to understand ICF-reactions. Some of these studies are summarized in an outstanding review by Gerschel [10]. It may, however, be pointed out that the existing models/theories fairly explain ICF data obtained at energies $\mathrm{E} \geq 10.5 \mathrm{MeV} / \mathrm{A}$ or so, but there is no theoretical model available to predict ICF at lower energies. Due to the unavailability of reliable theoretical model to predict low energy ICF, the experimental study of underlying dynamics is still an active area of investigation.

In view of the above, we have undertaken a programme to study the ICF-reaction dynamics in different projectiletarget combinations at low energies. The onset of ICF at slightly above barrier energies has been emphasized in the excitation function (EFs) measurements $[5,6]$, however, a clear existence of ICF at low energies has been demonstrated by measuring more than one linear momentum transfer components in the forward recoil ranges [7]. In addition to this, the unclear or ambiguous dependences of ICF on various entrance channel parameters have also been explored and contradicting dependences of the fraction of incomplete fusion have been reported [11-13]. Morgenstern et al. [13]correlated the ICF fraction with entrance channel mass asymmetry $\left(\mu_{A}\right)$. Recently, Singh et al. [5] introduced the importance of projectile structure in ProMass-systematics. Hence, in order to explore the lowenergy incomplete fusion and to find a consistent general systematics for low energy ICF reactions, which may support the SHE formation, measurements of excitation function for ${ }^{18} \mathrm{O},{ }^{13,12} \mathrm{C}+{ }^{159} \mathrm{~Tb}$ systems at energies $\approx 4-7 \mathrm{MeV} / \mathrm{A}$ and forward recoil ranges for ${ }^{12} \mathrm{C}+{ }^{159} \mathrm{~Tb}$ at three above barrier-energies have been performed.

\section{Experiments}

In order to ascertain above aspects, two kinds of experiments have been performed by our group at the Inter-University Accelerator Center (IUAC), New Delhi; (i) the experiments to measure "the excitation functions" of radio-nuclides populated during the interaction of ${ }^{18} \mathrm{O},{ }^{13,12} \mathrm{C}+{ }^{159} \mathrm{~Tb}$ systems at energies $\approx 4-7 \mathrm{MeV} / \mathrm{A}$, and (ii) the experiments to measure "the forward recoil ranges" of residues populated during the interaction of ${ }^{12} \mathrm{C}+{ }^{159} \mathrm{~Tb}$ at three widely different above barrier energies. Here, brief experimental details are given for the ready reference; however, the details are given in refs $[5,6]$. In these experiments the activation technique has been used. The irradiation of the samples have been carried out in the General Purpose Scattering Chamber having an in-vacuum transfer facility, which has been used to minimize the lapse time between the stop of the irradiation and beginning of the counting of the activity induced in the target-catcher assembly. The activities induced in the samples were recorded by counting each target along with the catcher foil, using a pre-calibrated HPGe $\gamma$-ray spectrometer. A $50 \mathrm{~Hz}$ pulser was used to determine the dead time. The
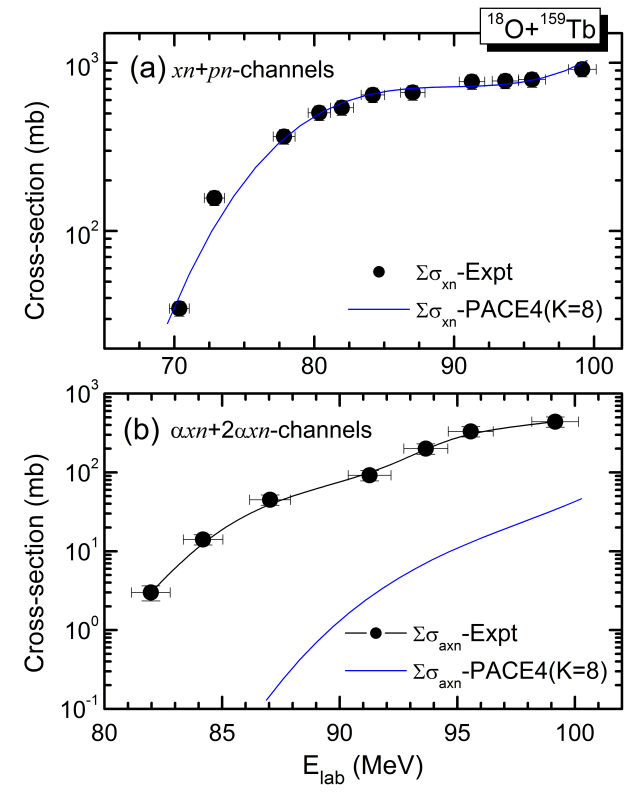

Figure 1. The experimentally measured and theoretically calculated EFs for (a) $x n+p x n$, and (b) $\alpha x n+2 \alpha x n$-channels populated in ${ }^{18} \mathrm{O}+{ }^{159} \mathrm{~Tb}$ interactions (for details see the text).

detector-sample separation was adjusted to keep the deadtime below $10 \%$ during the counting so as to minimize the pile up effects. The efficiency calibration of the detector in the specified geometry was carried out using a standard

${ }^{152} \mathrm{Eu}$ source of known strength at various source-detector separations. The characteristic $\gamma$-lines have been used to identify reaction products. Further, the decay curves of the identified reaction products have also been analyzed to confirm the identification. Nuclear data on radio-nuclides, such as the corresponding $\gamma$-ray abundances and half-lives were taken from ref [14]. The production cross sections of the reaction products have been determined using the standard formulation $[5,6]$. It may be pointed out that the errors in the measured production cross sections may arise due to $(i)$ the nonuniformity of target foils, (ii) fluctuations in the beam current, (iii) the uncertainty in geometry dependent efficiency of HPGe detector, and (iv) due to the dead time of the spectrometer. Detailed discussion on the error analysis is given elsewhere [5,6]. The overall errors including statistical errors are estimated to be $\geq 15 \%$, excluding the uncertainty in branching ratio, decay constant, etc.

\section{Interpretation of results}

\subsection{Excitation Functions (EFs)}

To understand the formation mechanism of reaction products, the experimentally measured excitation functions have been analyzed within the framework of statistical model code PACE4 [15] based on equilibrated CN-decay of Hauser-Feshbach theory. It may, however, be pointed out that the ICF and pre-equilibrium-emission (PEE) are not taken into consideration in this code. In this code, level density parameter $(\mathrm{a}=\mathrm{A} / \mathrm{K})$ is an important input parameter which affects the CF cross-sections and may be varied to match the experimental cross-sections. In the present 
system at studied energy range the parameter " $\mathrm{a}=\mathrm{A} / 8$ " has been found to reproduce satisfactorily, the experimental data for both the $x n$ and pxn-channels, which shows the production of these residues via CF processes. As a representative case, in Fig.1 (a) the sum of cross-sections for all experimentally measured $x n \& p x n$-channels for ${ }^{18} \mathrm{O}+{ }^{159} \mathrm{~Tb}$ system have been compared with that of corresponding theoretical calculations with physical justified set of parameters. The same set of parameters has been retained and used to check the production mechanism of $\alpha$ emitting channels, also. In Fig.1 (b), the sum of measured $\alpha x n$ and $2 \alpha x n$-channels for same system has been plotted and compared with theoretical calculations. As shown in Fig.1 (b), the measured cross-sections for $\alpha$-emitting channels are found to be significantly enhanced than theoretical predictions. The enhanced cross-section in case of $\alpha$-emitting channels point towards some physical effects which are not included in this code. It has already been mentioned that PACE4 do not take ICF, PEE into account and hence, this enhancement may be attributed as the contribution due to ICF-reaction mechanism.

\subsection{Forward Recoil Range Distributions (FRRDs)}

In heavy-ion interactions, the CF \& ICF processes may lead to the characteristic velocity distribution of the reaction products on the basis of linear momentum transfer from projectile to the target nucleus. As such, the distribution of measured yields of the populated reaction residues as a function of velocity and/or the range of residue in a stopping medium may give an insight into the reaction mechanism. Though the differences in the velocity and ranges of CF and ICF reaction products are not so significant, by using very thin catcher foils $\left(\approx \mu \mathrm{g} / \mathrm{cm}^{2}\right)$, it is possible to separate both kind of residues. Hence, in order to demonstrate the presence of ICF reactions in ${ }^{12} \mathrm{C}+{ }^{159} \mathrm{~Tb}$, the recoil ranges of residues have also been measured at three above barrier energies. The resulting normalized yields of different reaction products have been plotted against cumulative catcher foil thicknesses to obtain the differential recoil range distributions for the residues viz., ${ }^{168} \mathrm{Lu}(3 n)$, ${ }^{167} \mathrm{Lu}(4 n),{ }^{165} \mathrm{Lu}(6 n),{ }^{167} \mathrm{Yb}(p 3 n),{ }^{165} \mathrm{Tm}(\alpha 2 n),{ }^{163} \mathrm{Tm}$ $(\alpha 4 n),{ }^{161} \mathrm{Ho}(2 \alpha 2 n),{ }^{160} \mathrm{Ho}^{g}(2 \alpha 3 n)$ and ${ }^{160} \mathrm{Ho}^{m}(2 \alpha 3 n)$. In this paper, as a representative case to show different linear momentum transfer components in various $\mathrm{CF}$ and ICF processes the FRRDs for ${ }^{167} \mathrm{Lu}(4 n)$ and ${ }^{165} \mathrm{Tm}(\alpha 2 n)$ residues have been presented and shown in Figs.2(a-f). These figures clearly show the different momentum transfer components, depending on the fused mass of the projectile with the target nucleus. In the case of $4 n$-channel (Fig.2(a-c)), the measured FRRDs show only a single peak, at all the three bombarding energies, indicating only single linear momentum transfer component (a characteristic of the CF process) involved in the production of ${ }^{167} \mathrm{Lu}$ residues. Similarly, in case of $\alpha$-emitting channels, the residues ${ }^{165} \mathrm{Tm},{ }^{163} \mathrm{Tm},{ }^{161} \mathrm{Ho},{ }^{160} \mathrm{Ho}^{g}$ and ${ }^{160} \mathrm{Ho}^{m}$ are populated, respectively via $\alpha 2 n, \alpha 4 n, 2 \alpha 2 n$ and $2 \alpha 3 n$ channels. The observed FRRDs were resolved into more than one Gaussian peaks. As a representative case, the FRRDs for the residues, ${ }^{165} \mathrm{Tm}(\alpha 2 n)$, have been plotted at
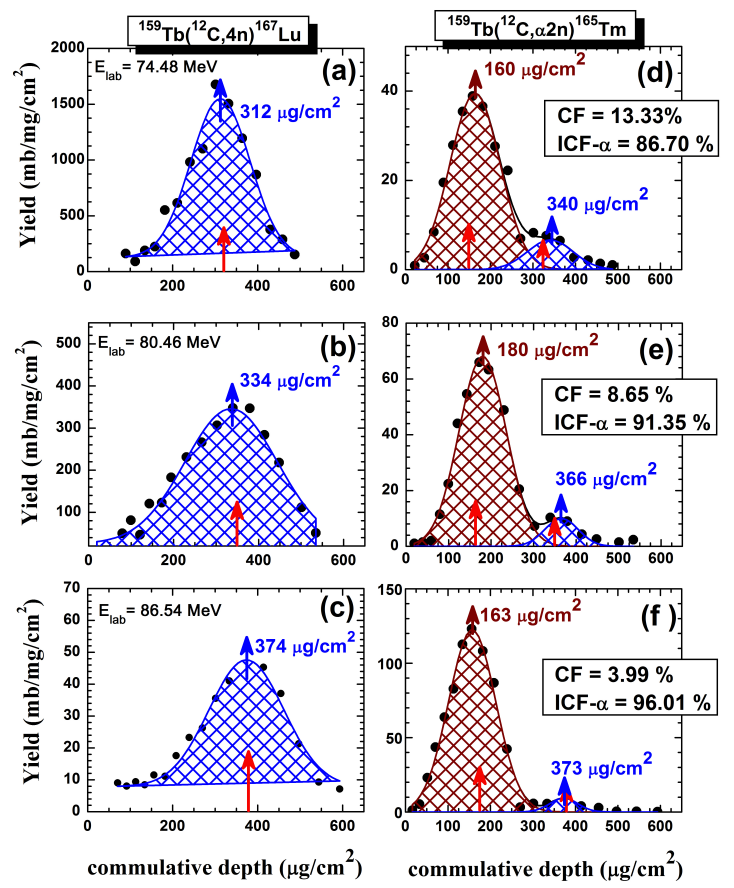

Figure 2. Typical FRRDs for ${ }^{167} \mathrm{Lu}$ and ${ }^{165} \mathrm{Tm}$ populated via $4 n$ and $\alpha 2 n$-channel, respectively. The red arrows are the theoretical most probabble ranges (for detail see text).

three different energies in Fig.2(d-f). As can be seen from this figure, the FRRDs may be fitted with two Gaussian peaks, one at $340 \pm 32,366 \pm 60$ and $373 \pm 65 \mu \mathrm{g} / \mathrm{cm}^{2}$ for three beam energies, indicating the complete momentum transfer events, however, another peak at lower cumulative depths correspond to the fusion of ${ }^{8} \mathrm{Be}$ (if ${ }^{12} \mathrm{C}$ is assumed to break-up into ${ }^{8} \mathrm{Be}+\alpha$ ) with ${ }^{159} \mathrm{~Tb}$ target nucleus. Similarly, the FRRDs for other $\alpha$-emitting channels indicating the presence of more than one linear momentum transfer component. It may be pointed out that, the neutron emission from the recoiling residues may change the energy/momentum of the recoiling nucleus, depending on the direction of emitted particles. This is reflected in the width (FWHM) of the experimentally measured recoil range distributions. The width may also arise because of the contribution from straggling effects. The most probable recoil ranges $\left(\mathrm{R}_{\text {theo }}\right)$ have also been theoretically calculated (shown in Fig.2 by red arrows); assuming that in the case of $\mathrm{CF}$, the incoming ion completely fuses with the target nucleus and transfers its total linear momentum to the fused system, which recoils to conserve the input linear momentum. The theoretically calculated and experimentally measured recoil ranges are found to agree reasonably well within the experimental errors (for details see ref. [6]).

\subsection{Incomplete fusion fraction}

It is evident from the analysis of EFs and FRRDs measurements that ICF-reactions contribute significantly to the production cross-section of $\alpha$-emitting channels at studied energies. Nevertheless, both the measurements of FRRDs and the EFs give nearly same $\mathrm{F}_{I C F}$ values for same system 


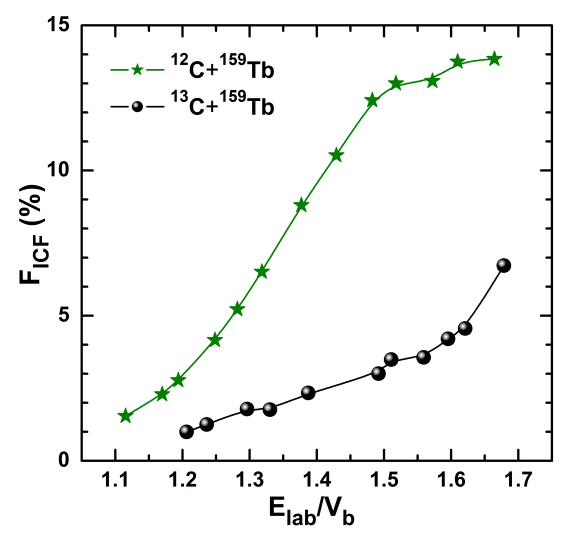

Figure 3. The comparison of $\mathrm{F}_{I C F}$ for ${ }^{13} \mathrm{C}+{ }^{159} \mathrm{~Tb}$ and ${ }^{12} \mathrm{C}+{ }^{159} \mathrm{~Tb}$ systems at constant $v_{\text {rel }}=0.053 \mathrm{c}$.

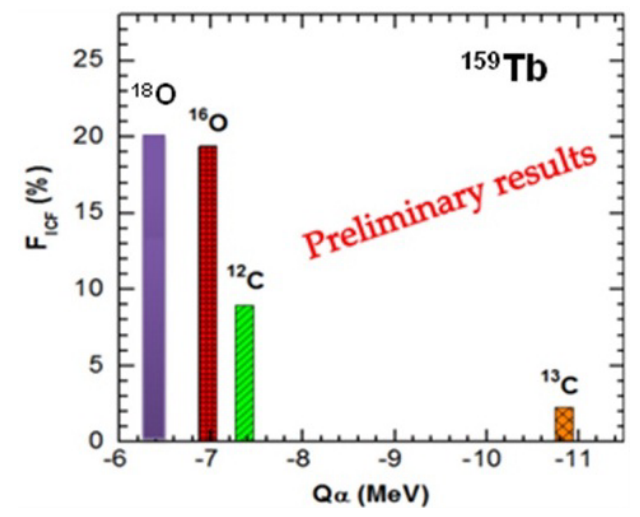

Figure 4. The comparison of incomplete fusion fraction for the studied systems ${ }^{18} \mathrm{O},{ }^{13,12} \mathrm{C}+{ }^{159} \mathrm{~Tb}$ with ${ }^{16} \mathrm{O}+{ }^{159} \mathrm{~Tb}[5]$.

which strengthen the measurements and indicate the selfconsistency of the data [6]. Further, the ICF contribution for all studied systems have been deduced using the prescription of Gomes et al., [4]. The ICF-contribution for individual channels has been deduced by subtracting $\mathrm{CF}$ cross-sections $\left(\Sigma \sigma_{C F}\right)$ from the experimentally measured total fusion cross sections $\left(\sigma_{T F}\right)$ at each studied energy. It is not out of place to mention that the $\sigma_{T F}$ has been corrected for the missing channels (which could not be measured experimentally) by their PACE4 values. Hence, the $\Sigma \sigma_{I C F}$ may be taken at least as the lower limit of ICFcontribution. The percentage $\mathrm{F}_{I C F}$, which is the measure of relative strength of ICF contribution to the total fusion, may be defined as, $\mathrm{F}_{I C F} \%=\left(\Sigma_{I C F} / \sigma_{T F}\right) \times 100$ have been deduced and the comparison of $\mathrm{F}_{I C F}$ for ${ }^{12} \mathrm{C}$ and $13 \mathrm{C}$ interactions with ${ }^{159} \mathrm{~Tb}$ target have been shown in Fig.3, where ${ }^{12} \mathrm{C}$ projectile having more ICF as compared to ${ }^{13} \mathrm{C}$ projectile, which shows a clear projectile dependency on ICF reactions.

\section{Remarks on the effect of projectile type}

In order to understand the effect of projectile on ICFreactions, the $\mathrm{F}_{I C F}$ for ${ }^{18,16} \mathrm{O},{ }^{13,12} \mathrm{C}+{ }^{159} \mathrm{~Tb}$ systems at a constant relative velocity $\left(v_{\text {rel }}=0.053\right)$ have been plotted as a function of $\mathrm{Q}_{\alpha}$-value of the projectile in Fig.4. This comparison of $\mathrm{F}_{I C F}$ for different projectiles on same target reveals a strong projectile dependence of low-energy incomplete fusion. It is clear from this figure, that the $\mathrm{F}_{I C F}$ is $\approx 18 \%$ larger for ${ }^{18} \mathrm{O}$ than ${ }^{13} \mathrm{C}$ as projectile on the same target ${ }^{159} \mathrm{~Tb}$, which can be understood by recently proposed alpha-Q-value systematics [6]. The more-negative $\mathrm{Q}_{\alpha}$-value for ${ }^{13} \mathrm{C}$ translates into the smaller breakup probability into constituent $\alpha$ clusters, resulting in a smaller ICF-fraction than for ${ }^{18} \mathrm{O}$ induced reactions. The present work strengthens the recently observed alpha-Q-value systematics [6] for strongly bound projectiles. It may, however, be pointed out that the systems studied in the present work are rather light, which may not cater to the requirement of synthesizing super heavy elements. However, a rich data set from medium to heavy targets may help to develop some systematics to understand the probability of involved reaction processes at these energies, which may be useful in the super heavy element research.

\section{Acknowledgements}

The authors thank to the Director, IUAC, New Delhi, India, for providing all the necessary facilities to carry out this work. One of authors A.Y. thanks the DST for providing support through Young Scientist Scheme under startup research grant.BPS, RP and DPS thank to DST and UGC for providing financial support.

\section{References}

[1] Yu. Ts. Oganessian et al., Nature (London) 400, 242 (1999), and references therein.

[2] M. Dasgupta et al., Nucl. Phys. A 787, 144-149 (2007).

[3] A. Diaz-Torres and I. J. Thompson, Phys. Rev. C 65, 024606 (2002); Phys. Rev. Lett. 98, 152701 (2007).

[4] P. R. S. Gomes et al, Phys. Rev. C 73, 064606 (2006); Phys. Lett. B 601, 20 (2004).

[5] Pushpendra P. Singh et al., Phys. Rev. C 77, 014607 (2008); Euro. Phys. J. A 34, 29-39 (2007).

[6] Abhishek Yadav et al., Phys. Rev. C 85, 034614 (2012); ibid 85, 064617 (2012); ibid 86, 014603 (2012) and references therein.

[7] D. P. Singh et al., Phys. Rev. C 81, 054607 (2010).

[8] T. Inamura et al., Phys. Lett. B 68, 51 (1977); Phys. Lett. B 84, 71 (1982); Phys. Rev. C 32, 1539 (1985).

[9] H. C. Britt and A. R. Quinton, Phys. Rev. 124, 877 (1961).

[10] C. Gerschel, Nucl. Phys. A 387, 297 (1982).

[11] L. F. Canto et al., Phys. Rev. C 58, 1107 (1998).

[12] M. Dasgupta et al., Phys. Rev. C 70, 024606 (2004).

[13] H. M. Morgenstern et al., Phys. Rev. Lett. 52, 1104 (1984); Z. Phys. A 313, 39 (1983).

[14] E. Browne and R. B. Firestone, Table of Radioactive Isotopes (Wiley, New York, 1986).

[15] A. Gavron, Phys. Rev. C 21, 230 (1980). 